\title{
Learning to Recover Reasoning Chains for Multi-Hop Question Answering via Cooperative Games
}

\author{
Yufei Feng ${ }^{\dagger, *}$, Mo Yu ${ }^{\ddagger}{ }^{*}$, Wenhan Xiong ${ }^{\diamond}$, Xiaoxiao Guo ${ }^{\ddagger}$, Junjie Huang ${ }^{\dagger}$, \\ Shiyu Chang ${ }^{\ddagger}$, Murray Campbell ${ }^{\ddagger}$, Michael Greenspan ${ }^{\dagger}$, Xiaodan Zhu $^{\dagger}$ \\ ${ }^{\dagger}$ Electrical and Computer Engineering \& Ingenuity Labs Research Institute, Queen’s University \\ $¥$ IBM Research \\ $\diamond$ UC Santa Barbara
}

\begin{abstract}
We extend the formats of explanations in interpretable NLP with the proposed entity-centric reasoning chains for multi-hop question answering. We also propose a cooperative game approach to learn to recover such explanations from weakly supervised signals, i.e., the question-answer pairs. We evaluate our task and method via newly created benchmarks based on two multi-hop datasets, HotpotQA and MedHop; and hand-labeled reasoning chains for the latter. The experiments demonstrate the effectiveness of our approach.
\end{abstract}

Keywords: Multi-hop Question Answering, Interpretability, Reasoning Chain, Model Cooperation

\section{Introduction}

Many recent work proposed to interpret neural predictions with various explanation formats, starting from feature importance with gradient information [1, 2], to locally fitted linear predictors [3, 4], subset of inputs sentences [5], semantic parses [6]. The different forms of explanations not only reveal information that are important for different tasks, but also determine the concrete interpretability algorithms.

We focus on the format of explanations that fit the recent popular task of multi-hop reasoning over texts [7,8], and develop an algorithm to derive such explanations from data with no humanannotated explanations. Specifically, taking multi-hop QA as an example, we propose a new form of explanations, entity-centric reasoning chains, which corresponds to a list of ordered passages and each pair of adjacent passages is connected with a linking entity (Figure 1). Compared existing tasks that take only evidence paragraphs as explanations [7], our form better fits the reasoning tasks via bringing two types of necessary structural information proved useful by recent work [9-13]: (1) the order of evidence paragraphs, which is crucial in logic reasoning over both symbols and texts [14]; and (2) how the evidence pieces are connected, which essentially defines the argument structures with linked entities, so as to formally represent $h o p s$.

To recover the reasoning chains without supervision, we propose a cooperative game approach to recovering the reasoning chains. Specifically, we start with a model, the Ranker, which selects a sequence of passages, with the constraints that each adjacent passage pair shares at least an entity, and the last passage contains the answer. Due to the lack supervised signals, the selection suffers from noise and ambiguity. To remedy this we introduce another model, the Reasoner, which predicts the exact linking entity from each passage that connects the next passage. The two models play a cooperative game and are rewarded when they find a consistent chain. In this way, we make the selections satisfy both the format constraints (i.e., ordered passages with connected adjacencies) and the semantic constraints (i.e., the connection to the next passage can be captured by a Reasoner model). Therefore, the selection can be less noisy.

The value of this research consists of two-folds. First, we propose a format of explanations that is powerful and easy-to-extend (e.g., refining the paragraphs in our chains to sentences or semantic parses). In contrast, the existing explanation formats are not representative enough for the increasing

*Equal Contribution.

feng.yufei@queensu.ca, yum@us.ibm.com 


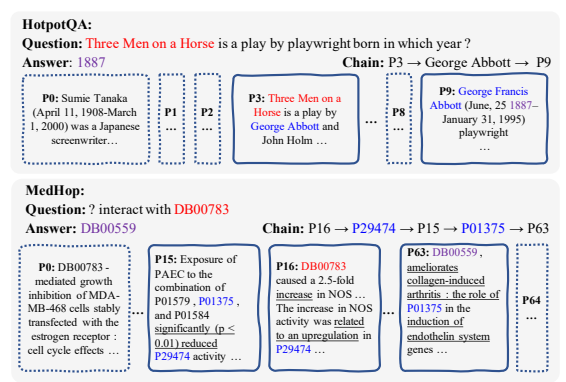

Figure 1. An example of reasoning chains in HotpotQA (2-hop) and MedHop (3-hop). HotpotQA provides only supporting passages $\left\{P_{3}, P_{9}\right\}$, without order and linking information.

complexity of NLP tasks and models, so works built on them lack faithfulness. Second, successfully recovering the reasoning chains eases the training of large NLP models. Recent progresses on multi-hop reasoning over texts $[12,13,15]$ heavily rely on the human-annotations of supporting evidence paragraphs/sentences and the applications of large pre-trained models [16-18]. These finergrained annotations allow the decomposition of model training into supervised sub-tasks. Without them, it is hard to apply the pre-trained models due to their memory inefficiency when handling long texts. However, human annotations are expensive and have limited and inflexible formats once collected. Automatic recovering evidence with flexible format can alleviate these problems.

We evaluate our method on datasets with different properties, HotpotQA and MedHop [19], that cover both 2-hop and 3-hop reasoning. We created labeled reasoning chains for both datasets. Experimental results demonstrate the significant advantage of our proposed approach.

\section{Task Definition}

Reasoning Chains. Examples of reasoning chains in HotpotQA and MedHop are shown in Figure 1. Formally, we aim at recovering the reasoning chain in the form of $\left(p_{1} \rightarrow e_{1,2} \rightarrow p_{2} \rightarrow e_{2,3} \rightarrow\right.$ $\cdots \rightarrow e_{n-1, n} \rightarrow p_{n}$ ), where each $p_{i}$ is a passage and each $e_{i, i+1}$ is an entity that connects $p_{i}$ and $p_{i+1}$, i.e., appearing in both passages. The last passage $p_{n}$ in the chain contains the correct answer. We say $p_{i}$ connects $e_{i-1, i}$ and $e_{i, i+1}$ for that it describes a relationship between the two entities.

Our Task. Given a QA pair $(q, a)$ and all its candidate passages $\mathcal{P}$, we can extract all possible candidate chains that satisfy the conditions mentioned above, denoted as $\mathcal{C}$. The goal of reasoning chain recovery is to extract the correct chains from all the candidates, given $q, a$ and $\mathcal{P}$ as inputs.

\section{Related Works}

Although there are recent interests on predicting reasoning chains for multi-hop QA [12, 13, 20], they all consider a fully supervised setting; i.e., annotated reasoning chains are available. Our work is the first to recover reasoning chains in a more general unsupervised setting, thus falling into the direction of denoising over distant supervised signals. From this perspective, the most relevant studies in the NLP field includes Wang, Yu, Guo, Wang, Klinger, Zhang, Chang, Tesauro, Zhou, and Jiang [21] and Min, Chen, Hajishirzi, and Zettlemoyer [22] for evidence identification in opendomain QA and Lei, Barzilay, and Jaakkola [5] and Perez, Karamcheti, Fergus, Weston, Kiela, and Cho [23] for rationale recovery.

\section{Method}

The task of recovering reasoning chains is essentially an unsupervised problem, as we have no access to annotated reasoning chains. Therefore, we resort to the noisy distant supervision signals from 


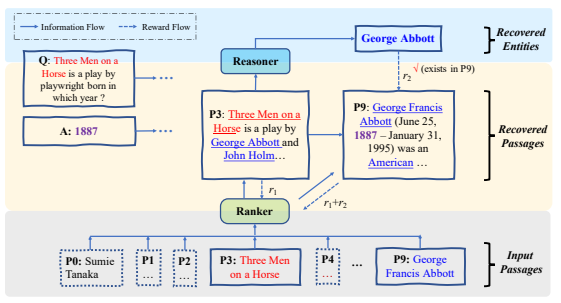

Figure 2. The cooperative Ranker and Reasoner are trained alternatively. The Ranker selects a passage $p$ at each step conditioned on the question $q$ and history selection, and receives reward $r_{1}$ if $p$ is evidence. Conditioned on $q$, the Reasoner predicts which entity in $p$ links to the next evidence passage. The Ranker receives extra reward $r_{2}$ if its next selection is connected by the entity predicted by the Reasoner.

question-answer pairs. We first propose a conditional selection model that optimizes the passage selection by considering their orders (Section 4.1). We then propose a cooperative Reasoner-Ranker game (Section 4.2) in which the Reasoner recovers the linking entities that point to the next passage Ranker selects. This encourages the Ranker to select chains with patterns that are easy for a model (Reasoner) to capture. Therefore, it helps our model de-noise the supervision signals and recover chains with entity information. Figure 2 illustrates our overall framework.

\subsection{Passage Ranking Model}

The key component of our framework is the Ranker model, which is provided with a question $q$ and $K$ passages $\mathcal{P}=\left\{p_{1}, p_{2} \ldots p_{K}\right\}$ from a pool of candidates, and outputs a chain of selected passages.

Passage Scoring. For each step of the chain, the Ranker estimates a distribution of the selection of each passage. To this end we first encode the question and passage with a 2-layer bi-directional GRU network, resulting in an encoded question $\boldsymbol{Q}=\left\{\overrightarrow{\boldsymbol{q}_{0}}, \overrightarrow{\boldsymbol{q}_{1}}, \ldots, \overrightarrow{\boldsymbol{q}_{N}}\right\}$ and $\boldsymbol{H}_{i}=\left\{\overrightarrow{\boldsymbol{h}_{i, 0}}, \overrightarrow{\boldsymbol{h}_{i, 1}}, \ldots, \overrightarrow{\boldsymbol{h}_{i, M_{i}}}\right\}$ for each passage $p_{i} \in \mathcal{P}$ of length $M_{i}$. Then we use the MatchLSTM model [24] to get the matching score between $\boldsymbol{Q}$ and each $\boldsymbol{H}_{i}$ and derive the distribution of passage selection $P\left(p_{i} \mid q\right)$. We denote $P\left(p_{i} \mid q\right)=\operatorname{MatchLSTM}\left(\boldsymbol{H}_{i}, \boldsymbol{Q}\right)$ for simplicity.

Conditional Selection. To model passage dependency along the chain of reasoning, we use a hard selection model that builds a chain incrementally. Provided with the $K$ passages, at each step $t$ the Ranker computes $P^{t}\left(p_{i} \mid \boldsymbol{Q}^{t-1}\right), i=0, \ldots, K$, which is the probability of selecting passage $p_{i}$ conditioned on the query and previous states representation $Q^{t-1}$. Then we sample one passage $p_{\tau}^{t}$ according to the predicted selection probability.

$$
\begin{array}{r}
p_{\tau}^{t}=\operatorname{Sampling}\left(\boldsymbol{P}^{t}\right), \boldsymbol{Q}^{t}=\operatorname{FFN}\left(\left[\boldsymbol{Q}^{t-1}, \tilde{\boldsymbol{m}}_{p_{\tau}}^{t}\right]\right) \\
\boldsymbol{P}^{t+1}\left(p_{i} \mid \boldsymbol{Q}^{t}\right)=\operatorname{MatchLSTM}\left(\boldsymbol{p}_{i}, \boldsymbol{Q}^{t}\right) .
\end{array}
$$

The first step starts with the original question $Q^{0}$. A feed-forward network is used to project the concatenation of query encoding and selected passage encoding $\tilde{\boldsymbol{m}}_{p_{\tau}}^{t}$ back to the query space, and the new query $\boldsymbol{Q}^{t+1}$ is used to select the next passage.

Reward via Distant Supervision. We use policy gradient [25] to optimize our model. As we have no access to annotated reasoning chains during training, the reward comes from distant supervision. Specifically, we reward the Ranker if a selected passage appears as the corresponding part of a distant supervised chain in $\mathcal{C}$. The model receives immediate reward at each step of selection.

In this paper we only consider chains consist of $\leq 3$ passages (2-hop and 3-hop chains). ${ }^{1}$ For the 2-hop cases, our model predicts a chain of two passages from the candidate set $\mathcal{C}$ in the form of $p_{h} \rightarrow e \rightarrow p_{t}$. Each candidate chain satisfies that $p_{t}$ contains the answer, while $p_{h}$ and $p_{t}$ contain a

${ }^{1}$ It has been show that $\leq 3$-hops can cover most real-world cases, such KB reasoning [26, 27]. 
shared entity $e$. We call $p_{h}$ the head passage and $p_{t}$ the tail passage. Let $\mathcal{P}_{T} / \mathcal{P}_{H}$ denote the set of all tail/head passages from $\mathcal{C}$. Our model receives rewards $r_{h}, r_{t}$ according to its selections:

$$
r_{t}=1.0 \Longleftrightarrow p_{t} \in \mathcal{P}_{\mathcal{T}}, r_{h}=1.0 \Longleftrightarrow p_{h} \in \mathcal{P}_{\mathcal{H}}
$$

For the 3-hop cases, we need to select an additional intermediate passage $p_{m}$ between $p_{h}$ and $p_{t}$. If we reward any $p_{m}$ selection that appears in the middle of a chain in candidate chain set $\mathcal{C}$, the number of feasible options can be very large. Therefore, we make our model first select the head passage $p_{h}$ and the tail passage $p_{t}$ independently and then select $p_{m}$ conditioned on $\left(p_{h}, p_{t}\right)$. We further restrict that each path in $\mathcal{C}$ must have the head passage containing an entity from $q$. Then the selected $p_{m}$ is only rewarded if it appears in a chain in $\mathcal{C}$ that starts with $p_{h}$ and ends with $p_{t}$ :

$$
\begin{aligned}
& r_{h}=1.0 \Longleftrightarrow p_{h} \in \mathcal{P}_{H}, r_{t}=1.0 \Longleftrightarrow p_{t} \in \mathcal{P}_{T} \\
& r_{m}=1.0 \Longleftrightarrow \text { path }\left(p_{h}, p_{m}, p_{t}\right) \in \mathcal{C}
\end{aligned}
$$

\subsection{Cooperative Reasoner}

To alleviate the noise in the distant supervision signal $\mathcal{C}$, in addition to the conditional selection, we further propose a cooperative Reasoner model, also implemented with the MatchLSTM architecture, to predict the linking entity from the selected passages. Given the question embed$\operatorname{ding} \boldsymbol{Q}^{r}=\left\{\overrightarrow{\boldsymbol{q}_{0}^{r}}, \overrightarrow{\boldsymbol{q}_{1}^{r}}, \ldots, \boldsymbol{q}_{N}^{\vec{r}}\right\}$ and the input passage embedding $\boldsymbol{H}^{r}=\left\{\overrightarrow{\boldsymbol{h}_{0}^{r}}, \overrightarrow{\boldsymbol{h}_{1}^{r}}, \ldots, \boldsymbol{h}_{M}^{\vec{r}}\right\}$ of $p$, the Reasoner predicts the probability of each entity in the passage being the linking entity of the next passage in the chain. We use a reader model similar to [7] as our Reasoner network.

For each token $e_{k}, k=0,1, \ldots, M$ represented by $h_{p, k}^{r}$ at the corresponding location, we have:

$$
P^{r}\left(e_{k} \mid p\right)=\left\{\begin{array}{ll}
g\left(\boldsymbol{h}_{p, k}^{r}\right), & \text { if } e_{k} \text { is a named entity } \\
0, & \text { otherwise }
\end{array} .\right.
$$

where $g$ is the classification layer, softmax is applied across all entities to get the probability. We denote the computation above as $P^{r}\left(e_{k} \mid p\right)=$ MatchLSTM.Reader $\left(e_{k}, p\right)$ for simplicity.

\subsection{Alternative Training}

Intuitively, when the Ranker makes more accurate passage selections, the Reasoner will work with less noisy data and thus is easier to succeed. Specifically, the Reasoner learns to extract the linking entity from chains selected by a well-trained Ranker, and it benefits the Ranker training by providing extra rewards. Taking 2-hop as an example, we train the Ranker and Reasoner alternatively as a cooperative game:

Reasoner Step: Given the first passage $p_{t}{ }^{2}$ selected by the trained Ranker, the Reasoner predicts the probability of each entity $e$ appearing in $p_{t}$. The Reasoner is trained with the cross-entropy loss:

Ranker Step: Given the Reasoner's top-1 predicted linking entity $e$, the reward for Ranker at the $2^{\text {nd }}$ step is defined as:

$$
\begin{aligned}
\boldsymbol{P}\left(e \mid p_{t}, q\right) & =\text { MatchLSTM_Reader }\left(\boldsymbol{H}_{p_{t}}, \boldsymbol{q}\right) \\
y_{e} & =\left\{\begin{array}{l}
1, \text { if } e \in p_{h} \\
0, \text { otherwise }
\end{array} \quad r_{h}=\left\{\begin{array}{l}
1, \text { if } p_{h} \in \mathcal{P}_{H} \\
1+r, \text { if } e \in p_{h}, p_{h} \in \mathcal{P}_{H} \\
0, \text { otherwise }
\end{array}\right.\right.
\end{aligned}
$$

\footnotetext{
${ }^{2}$ The same method holds for selecting $p_{h}$ first. Section 5 shows starting from the answer is empirically better.
} 
Table 1. Reasoning Chain selection results.

\begin{tabular}{lcc}
\hline Model & HotpotQA & MedHop \\
\hline Random & $40.3 \%$ & $56.0 \%$ \\
Dist. Sup. MatchLSTM & $74.0 \%$ & $59.3 \%$ \\
Conditional Selection & $84.7 \%$ & $59.3 \%$ \\
Cooperative Game & $87.2 \%$ & $62.6 \%$ \\
\hline
\end{tabular}

Table 2. Ablation test on HotpotQA.

\begin{tabular}{lcc}
\hline Model - Hotpot & Head/Tail & EM \\
\hline Cond. Selection (Head to Tail) & $80.7 / 95.0 \%$ & $77.1 \%$ \\
Cond. Selection (Tail to Head) & $88.1 / 96.2 \%$ & $84.7 \%$ \\
$\quad$ + Cooperative Reasoner & $90.1 / 96.7 \%$ & $87.2 \%$ \\
\hline
\end{tabular}

The extension to 3-hop cases is straightforward; the only difference is that the Reasoner reads both the selected $p_{h}$ and $p_{t}$ to output two entities. The Ranker receives one extra reward if the Reasoner picks the correct linking entity from $p_{h}$, so does $p_{t}$.

\section{Experiments}

We evaluate our reasoning chain selection model on HotpotQA bridge type questions and on the MedHop dataset. In HotpotQA, the entities are pre-processed Wiki anchor link objects and in MedHop they are drug/protein database identifiers. For HotpotQA, two supporting passages are provided along with each question. We ignore the support annotations during training and use them to create ground truth on development set: following [14], we determine the order of passages according to whether a passage contains the answer. We discard ambiguous instances. For MedHop, there is no evidence annotated. Therefore we created a new evaluation dataset by manually annotating the correct paths for part of the development set: we first extract all candidate paths in form of passage triplets $\left(p_{h}, p_{m}, p_{t}\right)$, such that $p_{h}$ contains the query drug and $p_{t}$ contains the answer drug, and $p_{h} / p_{m}$ and $p_{m} / p_{t}$ are connected by shared proteins. We label a chain as positive if all the drug-protein or protein-protein interactions are described in the corresponding passages. Note that the positive paths are not unique for a question. We compare our model with (1) random baseline, which randomly selects a valid candidate chain from the distant supervision chain set $\mathcal{C}$; and (2) distant supervised MatchLSTM, which uses the same base model as ours but scores and selects the passages independently. We use accuracy as our evaluation metric.

HotpotQA. We first evaluate on the 2-hop HotpotQA task. Our best performed model first selects the tail passage $p_{t}$ and then the head passage $p_{h}$, because the number of candidates of tail is smaller ( $\sim 2$ per question). Table 1 shows the results. First, training a ranker with distant supervision performs significantly better than the random baseline, showing that the training process itself has a certain degree of denoising ability to distinguish the more informative signals from distant supervision labels. By introducing additional inductive bias of orders, the conditional selection model further improves with a large margin. Finally, our cooperative game gives the best performance, showing that a trained Reasoner has the ability of ignoring entity links that are irrelevant to the reasoning chain. Table 2 shows the effect of selecting directions and the methods' recall on head passages and tail passages. The latter is evaluated on a subset of bridge-type questions in HotpotQA which has no ambiguous support annotations in passage orders; i.e., among the two human-labeled supporting passages, only one contains the answer and thus must be a tail. The results show that selecting tail first performs better. The cooperative game mainly improves the head selection.

MedHop. Results in table 1 show that recovering chains from MedHop is a much harder task: first, the large number of distant supervision chains in $\mathcal{C}$ introduce too much noise so the Distant Supervised Ranker improves only 3\%; second, the dependent model leads to no improvement because $\mathcal{C}$ is strictly ordered given our data construction. Our cooperative game manages to remain effective and gives further improvement. 


\section{Conclusions}

In this paper we propose the problem of recovering reasoning chains in multi-hop QA from weak supervision signals. Our model adopts an cooperative game approach where a ranker and a reasoner cooperate to select the most confident chains. Experiments on the HotpotQA and MedHop benchmarks show the effectiveness of the proposed approach.

\section{References}

[1] J. Li, X. Chen, E. Hovy, and D. Jurafsky. "Visualizing and Understanding Neural Models in NLP”. In: Proceedings of the NAACL-HLT. 2016, pp. 681-691.

[2] M. Sundararajan, A. Taly, and Q. Yan. "Axiomatic attribution for deep networks". In: Proceedings of the 34th International Conference on Machine Learning-Volume 70. JMLR. org. 2017, pp. 3319-3328.

[3] M. T. Ribeiro, S. Singh, and C. Guestrin. "Why should I trust you?: Explaining the predictions of any classifier". In: Proceedings of the 22nd ACM SIGKDD international conference on knowledge discovery and data mining. ACM. 2016, pp. 1135-1144.

[4] D. Alvarez-Melis and T. S. Jaakkola. "Towards Robust Interpretability with Self-Explaining Neural Networks”. In: arXiv preprint arXiv:1806.07538 (2018).

[5] T. Lei, R. Barzilay, and T. Jaakkola. "Rationalizing Neural Predictions". In: Proceedings of the 2016 Conference on Empirical Methods in Natural Language Processing. 2016, pp. 107-117.

[6] J. Andreas, M. Rohrbach, T. Darrell, and D. Klein. "Learning to Compose Neural Networks for Question Answering". In: Proceedings of NAACL-HLT. 2016, pp. 1545-1554.

[7] Z. Yang, P. Qi, S. Zhang, Y. Bengio, W. W. Cohen, R. Salakhutdinov, and C. D. Manning. "HotpotQA: A Dataset for Diverse, Explainable Multi-hop Question Answering". In: Proc. of EMNLP 2018. 2018.

[8] Y. Yao, D. Ye, P. Li, X. Han, Y. Lin, Z. Liu, Z. Liu, L. Huang, J. Zhou, and M. Sun. "DocRED: A Large-Scale Document-Level Relation Extraction Dataset". In: Proceedings of ACL 2019. 2019.

[9] N. De Cao, W. Aziz, and I. Titov. "Question Answering by Reasoning Across Documents with Graph Convolutional Networks". In: Proceedings of NAACL-HLT 2019. 2019.

[10] S. Kundu, T. Khot, and A. Sabharwal. "Exploiting Explicit Paths for Multi-hop Reading Comprehension". In: Proceedings of ACL 2019. 2019.

[11] Y. Xiao, Y. Qu, L. Qiu, H. Zhou, L. Li, W. Zhang, and Y. Yu. "Dynamically Fused Graph Network for Multi-hop Reasoning”. In: Proceedings of ACL 2019. 2019.

[12] M. Ding, C. Zhou, Q. Chen, H. Yang, and J. Tang. "Cognitive Graph for Multi-Hop Reading Comprehension at Scale". In: Proceedings of ACL 2019. 2019.

[13] A. Asai, K. Hashimoto, H. Hajishirzi, R. Socher, and C. Xiong. "Learning to Retrieve Reasoning Paths over Wikipedia Graph for Question Answering”. In: arXiv preprint arXiv:1911.10470 (2019).

[14] H. Wang, M. Yu, X. Guo, R. Das, W. Xiong, and T. Gao. "Do Multi-hop Readers Dream of Reasoning Chains?” In: arXiv preprint arXiv:1910.14520 (2019).

[15] Y. Nie, S. Wang, and M. Bansal. "Revealing the Importance of Semantic Retrieval for Machine Reading at Scale”. In: Proceedings of EMNLP 2019. 2019.

[16] M. Peters, M. Neumann, M. Iyyer, M. Gardner, C. Clark, K. Lee, and L. Zettlemoyer. "Deep Contextualized Word Representations". In: Proceedings of NAACL-HLT. 2018.

[17] A. Radford, K. Narasimhan, T. Salimans, and I. Sutskever. Improving language understanding with unsupervised learning. Tech. rep. Technical report, OpenAI, 2018.

[18] J. Devlin, M.-W. Chang, K. Lee, and K. Toutanova. "BERT: Pre-training of Deep Bidirectional Transformers for Language Understanding”. In: Proceedings of NAACL-HLT 2019. 2019.

[19] J. Welbl, P. Stenetorp, and S. Riedel. "Constructing datasets for multi-hop reading comprehension across documents". In: Transactions of the Association for Computational Linguistics 6 (2018), pp. 287-302.

[20] J. Chen, S.-t. Lin, and G. Durrett. "Multi-hop Question Answering via Reasoning Chains". In: arXiv preprint arXiv:1910.02610 (2019).

[21] S. Wang, M. Yu, X. Guo, Z. Wang, T. Klinger, W. Zhang, S. Chang, G. Tesauro, B. Zhou, and J. Jiang. "R3: Reinforced Ranker-Reader for Open-Domain Question Answering". In: Proc. of AAAI 2018. 2018.

[22] S. Min, D. Chen, H. Hajishirzi, and L. Zettlemoyer. "A Discrete Hard EM Approach for Weakly Supervised Question Answering”. In: Proceedings of EMNLP 2019. 2019.

[23] E. Perez, S. Karamcheti, R. Fergus, J. Weston, D. Kiela, and K. Cho. "Finding Generalizable Evidence by Learning to Convince Q\&A Models”. In: Proceedings of EMNLP 2019. 2019.

[24] S. Wang and J. Jiang. "Learning Natural Language Inference with LSTM". In: Proceedings of NAACLHLT 2016. 2016, pp. 1442-1451. 
[25] R. J. Williams. "Simple statistical gradient-following algorithms for connectionist reinforcement learning”. In: Machine learning 8.3-4 (1992), pp. 229-256.

[26] W. Xiong, T. Hoang, and W. Y. Wang. "DeepPath: A Reinforcement Learning Method for Knowledge Graph Reasoning". In: Proceedings of EMNLP 2017. 2017.

[27] R. Das, S. Dhuliawala, M. Zaheer, L. Vilnis, I. Durugkar, A. Krishnamurthy, A. Smola, and A. McCallum. "Go for a Walk and Arrive at the Answer: Reasoning Over Paths in Knowledge Bases using Reinforcement Learning”. In: Proceedings of ICLR 2018. 2018. 